\title{
Barreiras às intervenções relacionadas à saúde do trabalhador do setor saúde no Brasil
}

\author{
Érica Lui Reinhardt ${ }^{1}$ e Frida Marina Fischer ${ }^{2}$
}

Como citar Reinhardt EL, Fischer FM. Barreiras às intervenções relacionadas à saúde do trabalhador do setor saúde no Brasil. Rev Panam Salud Publica. 2009;25(5):411-7.

RESUMO Objetivo. Identificar na literatura situações que possam impedir ou prejudicar as ações de prevenção de acidentes e doenças ou de promoção da saúde de trabalhadores do setor saúde.

Método. Foi realizada uma revisão da literatura utilizando a base SciELO para o período de 1967 a 2008, complementada por busca na base PubMed para o período de 1950 a 2008. Os seguintes termos foram utilizados para identificar artigos em português, inglês e espanhol: trabalho, trabalhador, ocupacional, riscos, doenças, ergonomia, capacidade para o trabalho, qualidade de vida, organização, acidentes, condições de trabalho, intervenção e administração. Foram selecionados artigos sobre prevenção de doenças e acidentes e sobre promoção da saúde no trabalho em serviços de saúde latino-americanos. Também foram selecionados artigos sobre intervenções em ambientes de trabalho no setor saúde.

Resultados. Foram identificadas as seguintes situações desfavoráveis: programas de intervenção sem boa base teórica e não integrados à gestão do serviço como um todo; falhas em avaliar a eficácia das intervenções; vigilância da saúde restrita a doenças e agravos específicos; falta de compromisso da gestão com as intervenções; falhas na comunicação; falta de participação e controle por parte dos trabalhadores sobre o ambiente de trabalho; e programas e intervenções baseados exclusivamente na mudança comportamental dos trabalhadores.

Conclusões. A literatura mostra que todas as barreiras citadas afetam tanto a melhoria do estado de saúde dos trabalhadores em saúde quanto a sua capacidade para o trabalho.

Palavras chave Revisão, setor de assistência à saúde, saúde do trabalhador, pessoal de saúde, Brasil.

Uma análise do conjunto das pesquisas sobre a relação entre saúde e trabalho mostra que a preocupação com a saúde dos trabalhadores no setor saúde foi mais tardia do que em outras categorias profissionais. No Brasil, Santana (1) relata

\footnotetext{
1 Fundacentro, Coordenação de Higiene do Trabalho, São Paulo (SP), Brasil. Correspondência: Rua Capote Valente 710, CEP 05409-002, São Paulo, SP, Brasil. E-mail: erica.reinhardt@fundacentro. gov.br

2 Universidade de São Paulo, Faculdade de Saúde Pública, Departamento de Saúde Ambiental, São Paulo (SP), Brasil. E-mail: fmfische@usp.br
}

que, embora a primeira tese localizada sobre saúde do trabalhador seja de 1951, foi somente na década de 1980 que surgiu a primeira tese abordando trabalhadores da saúde. A partir de então, esse atraso foi compensado: na década de 1980 e na seguinte, nenhuma categoria profissional recebeu tanta atenção, com pouco mais de $15 \%$ de todas as teses e dissertações entre 2000 e 2004 dedicadas a esse grupo. Vários aspectos foram estudados: rotatividade, riscos químicos e biológicos, acidentes de trabalho, acidentes com perfurocortantes, ergonomia, sintomas e doenças osteomusculares, hepatite B e, sobretudo, estresse e saúde mental (1).

Esse crescimento na produção acadêmica acompanhou o surgimento da AIDS e a sua consolidação como epidemia. Até então, nenhuma outra doença havia suscitado tanto debate quanto à segurança dos profissionais de saúde, apesar de já se conhecerem os riscos de transmissão de muitos outros agentes causadores de doenças graves (2). Os primeiros 2 anos da epidemia de AIDS ensejaram a formulação de precauções 
com sangue e fluidos com sangue visível. Em 1984, 1 ano após a descoberta do HIV, ocorreu o primeiro relato de transmissão em ambiente de trabalho. Em 1987, os Centers for Disease Control and Prevention (CDC), nos Estados Unidos, tomaram conhecimento de outros três casos, recomendando, no mesmo ano, a adoção das "precauções universais" com sangue ou outros líquidos corpóreos por parte de todos os profissionais de saúde (2).

Esse período, incluindo a década de 1990, coincidiu ainda com modificações no sistema de saúde no Brasil e com a criação do Sistema Único de Saúde (SUS). Essa década também foi marcada pelo debate em torno da reforma do Estado na América Latina, dentro do contexto da globalização e da internacionalização da economia $(3,4)$, que propiciaram transformações na dinâmica dos mercados de trabalho em geral e, particularmente, nas profissões da área da saúde. Nesse setor, aumentaram as exigências quanto ao ingresso de pessoal, principalmente o de apoio; intensificou-se ainda a participação de profissionais com maior formação gerencial e de trabalhadores terceirizados, inclusive de profissionais de nível superior, na forma de cooperativas médicas e organizações de enfermagem para atenção domiciliar ou reabilitação (4).

No Brasil, tanto a epidemia de AIDS quanto as transformações no trabalho reforçaram o debate sobre os trabalhadores da saúde, levando entidades representativas de várias categorias a reivindicar legislação específica para o setor. Uma das mudanças na legislação foi o surgimento da Norma Regulamentadora 32 (NR-32) (5), do Ministério do Trabalho e Emprego, especificamente sobre o trabalho em saúde. Também ocorreram alterações na legislação dos Ministérios da Saúde e da Previdência e Assistência Social, com o reconhecimento explícito de doenças como tuberculose, hepatites virais e AIDS como doenças do trabalho, e também o estabelecimento do nexo entre o trabalho em hospitais e agravos como transtornos mentais, doenças do sistema nervoso, doenças osteomusculares e outros $(6,7)$. Porém, apesar de essas mudanças subsidiarem a formulação de programas de prevenção de acidentes e doenças em serviços de saúde, certos aspectos relativos à promoção da saúde desses trabalhadores não foram adequadamente abordados pela le- gislação, por exemplo aqueles relacionados à valorização do trabalho e das carreiras, à modificação das relações de trabalho e da forma de gestão desses serviços e à ampliação do espaço para participação ativa de todos os trabalhadores.

Além disso, muitas vezes os avanços no conhecimento e na legislação permaneceram como meras declarações de intenções, e o estabelecimento, na prática, de ações de prevenção ou de promoção da saúde continua sendo uma realidade distante. Assim, o objetivo deste trabalho foi examinar a literatura a fim de identificar elementos, situações ou contextos que poderiam impedir ou prejudicar a implantação de ações e intervenções dirigidas à saúde do trabalhador do setor saúde.

\section{MÉTODO}

Foram feitas buscas em português, espanhol e inglês na base de dados SciELO (www.scielo.org) usando os seguintes termos, isoladamente ou em combinação: trabalho (trabajo/work), trabalhador (trabajador ou personal de salud/ worker ou health personnel), ocupacional (ocupacional/occupational), riscos (riesgos/risks), doenças (enfermedades ou molestias/diseases), ergonomia (ergonomía/ergonomics), capacidade para o trabalho (capacidad para el trabajo/ work ability), qualidade de vida (calidad de vida/quality of life), organização (organización/organization), acidentes (accidentes/accidents), condições de trabalho (condiciones de trabajo/work conditions), intervenção (intervención/ intervention) e administração (administración/administration). A busca abrangeu todo o período coberto pela base de dados, de 1967 a 2008.

Também foram pesquisadas as publicações relativas ao setor saúde disponíveis na Biblioteca Virtual em Saúde (www.bvs.br/php/index.php) e livros texto sobre saúde ocupacional, políticas de saúde e correlatos. Foi feita uma seleção dos artigos sobre prevenção de doenças e acidentes e sobre promoção da saúde no trabalho em serviços de saúde latino-americanos, resultando em uma quantidade pequena de artigos com foco no diagnóstico das condições de trabalho e saúde dos trabalhadores ou na avaliação dos resultados das intervenções realizadas. Por essa razão, utilizaram-se também artigos sobre intervenções em ambientes de trabalho no setor saúde obtidos após busca na base de dados PubMed (www.ncbi.nlm.nih.gov) englobando o período de 1950 a 2008, que correspondia ao total de anos cobertos por esta base de dados no momento do estudo. Como complementação adicional, os mesmos termos foram usados para pesquisar outros artigos relacionados à saúde do trabalhador em ambas as bases e abrangendo os mesmos períodos já citados, porém não restritos ao setor saúde.

\section{RESULTADOS}

Na base de dados SciELO foram localizados 88 artigos sobre a saúde e as condições de trabalho dos trabalhadores no setor saúde. Setenta e cinco desses estudos apresentavam um diagnóstico da segurança e das condições de trabalho e de saúde desses trabalhadores, fornecendo subsídios para a elaboração e o desenvolvimento de ações e intervenções. Em somente 13 trabalhos foi feita uma avaliação dos resultados desse tipo de intervenção, o que pode estar refletindo a dificuldade de implantação e execução dessas ações.

Além disso, nos 88 trabalhos localizados na SciELO, foi possível perceber duas maneiras de abordar a saúde dos trabalhadores. Uma delas enfocava a prevenção, especialmente de acidentes e doenças relacionados diretamente com as condições ou fatores de risco do ambiente de trabalho. Na outra abordagem houve maior ênfase em conceitos da promoção da saúde dos trabalhadores. Essa abordagem buscava correlacionar a organização e os processos de trabalho, assim como situações presentes fora do ambiente laboral, com a ocorrência de desvios no estado de saúde, com possíveis impactos sobre a qualidade de vida e a satisfação desses trabalhadores com o seu trabalho ou sua capacidade para desempenhá-lo.

Enquanto a promoção da saúde propõe uma participação do trabalhador na busca pela saúde, a prevenção de acidentes e doenças orienta-se por um enfoque unidirecional, onde a tomada de decisão é feita quase que somente por especialistas. Entretanto, as duas abordagens são necessárias e complementares para um bom programa de saúde do trabalhador, pois cada uma possui especificidades e resultados diferentes. Um programa voltado para a prevenção de acidentes e doenças seria mais específico e particu- 
lar, mais ágil e com resultados mais imediatos. Já um programa com foco na promoção da saúde seria mais abrangente e geral, mais demorado e com resultados em prazos maiores.

Dos estudos identificados, 53 adotavam a abordagem da prevenção de acidentes e doenças, enfocando, entre outros, riscos químicos (8), acidentes com perfurocortantes $(9,10)$ e doenças do sistema osteomuscular (11). Exemplos dos 35 estudos que abordavam a promoção da saúde são os artigos sobre o trabalho da enfermagem em um hospital universitário (12), sobre as condições de trabalho e saúde de médicos (13) e sobre a terceirização de trabalhadores de limpeza hospitalar (14).

\section{Limitações dos programas de prevenção de acidentes e doenças}

Quando mal conduzida, a prevenção de acidentes e doenças pode se tornar excessivamente reducionista e centralizada nos desvios da situação normal, expressos como doenças relacionadas ao trabalho, incidentes e acidentes. Nesse caso, o contexto dos desvios é ignorado e se focaliza apenas o instante de sua ocorrência e as circunstâncias imediatas, levando à concepção de que ele é um evento simples, com uma ou poucas causas encadeadas de modo linear e determinístico. Prevalece a ideia de que os desvios decorrem de falhas dos trabalhadores ou de desrespeito à norma ou prescrição de segurança, com a consequente responsabilização desses mesmos trabalhadores por seu agravo ou doença (15).

Infelizmente, programas mal conduzidos de prevenção de acidentes e doenças ocupacionais também podem ocorrer em serviços de saúde, como no caso de programas implementados para prevenir a transmissão de patógenos sanguíneos (16). Muitas vezes, tais programas são centrados nas recomendações conhecidas como precauções-padrão, que até recentemente baseavam-se quase que exclusivamente no comportamento dos trabalhadores e em sua adesão a elas. Anteriormente denominadas precauções universais, essas medidas incluem o uso de uma grande variedade de equipamentos de proteção individual, higienização frequente das mãos e manipulação prudente de perfurocortantes (17).

No entanto, as precauções-padrão falham, e cabe perguntar o porquê. Segundo
Osterman (16), isso ocorre porque, na maioria dos ambientes de trabalho, as abordagens de segurança e prevenção têm sua ênfase na adesão ativa de cada trabalhador. As falhas podem ocorrer por razões técnicas, como quando os equipamentos de proteção interferem com o procedimento. Também podem ser administrativas, como na situação em que o descarte correto de agulhas é impedido porque os recipientes estão cheios. Algumas falhas seriam devidas à capacitação inadequada, embora a literatura demonstre que a execução de tarefas repetitivas e rotineiras que requerem muita vigilância ou concentração torna os erros inevitáveis, mesmo entre os trabalhadores mais bem capacitados. Entretanto, a razão mais importante é a dificuldade de modificar o conhecimento, o comportamento e as atitudes individuais (16). Uma ênfase exagerada no envolvimento individual ativo com as medidas de prevenção desconsidera as limitações humanas (16). E, quando as precauções-padrão falham, pode haver a responsabilização da vítima, ou pior, emergir a noção de que tais eventos são inevitáveis - como demonstra um estudo realizado no Brasil, onde os trabalhadores expostos acidentalmente a materiais corporais humanos relataram que o acidente simplesmente aconteceu, como se fosse inevitável ou inerente à atividade (18). Outras causas apontadas incluíram falta de atenção (18-20), descuido ou uso incorreto dos equipamentos de proteção individual, indicando que os próprios trabalhadores se assumem como responsáveis e culpados pelo acidente (18, 20).

Atribuir a causa do acidente a uma ação do trabalhador ou à natureza intrínseca do trabalho pode impedir ou dificultar uma investigação mais aprofundada dos fatores, das situações e do contexto que levam ao acidente e, consequentemente, o desenvolvimento de estratégias para impedir ou minimizar essas ocorrências. Uma forma de evitar essa situação é não restringir as ações de vigilância somente à vigilância médica, voltada para o monitoramento de doenças, o atendimento clínico e o acompanhamento de trabalhadores expostos, isto é, baseada em eventos já ocorridos. Essa vigilância deve, pelo contrário, continuamente identificar e detectar as mudanças nos vários determinantes da saúde dos trabalhadores, sejam eles individuais, ambientais, psicossociais ou organizacionais, de maneira a propor recomen- dações de prevenção e controle desses determinantes antes que ocasionem prejuízos à saúde dos trabalhadores (21).

Frente aos acidentes, muitas vezes são oferecidos cursos ou treinamentos para os trabalhadores, no intuito de obter maior adesão às medidas de prevenção. Paradoxalmente, porém, essas ações podem não aumentar a adesão de forma expressiva (22-25). Não que tais informações ou treinamentos sejam desnecessários, ao contrário, são determinantes para a adesão dos trabalhadores às medidas de prevenção (26). Porém, se observa que o mero conhecimento das medidas de prevenção e a formação centrada em aspectos técnicos pode não corresponder a uma maior adesão, na prática, a essas medidas $(22-24,27)$, sendo que o gerenciamento dos riscos ligados a perfurocortantes requer a combinação de diferentes estratégias de intervenção, incluindo o uso de perfurocortantes com dispositivos de segurança (22).

Para aumentar a adesão, há sugestões na literatura de que esses cursos e treinamentos deveriam ser periódicos, dirigidos às necessidades específicas de um grupo ou departamento e elaborados de modo a obter uma mudança permanente na prática cotidiana de trabalho, que inclua a valorização e a integração efetivas das medidas de prevenção $(26,28)$. Além da conscientização e da educação permanente dos trabalhadores, também é necessário proporcionar oportunidades para reflexão, discussões críticas e atualizações e condições materiais e ambientais para um trabalho seguro, controlando as situações que interferem com a adesão, como a carga de trabalho, a percepção do risco envolvido e a disponibilidade de equipamentos e vestimentas de proteção e tempo para a execução das atividades $(28,29)$.

Por fim, para que os programas de prevenção de acidentes e doenças sejam mais efetivos, as evidências sugerem que a organização deve estar realmente comprometida com a segurança e a saúde de seus trabalhadores e que esse compromisso deve ser visível e permear as tomadas de decisão gerenciais e as normas, expectativas, práticas, políticas e os procedimentos de segurança existentes (30). Em outras palavras, os programas de prevenção de acidentes e doenças devem ser considerados como integrantes da gestão dos serviços de saúde, e não como programas à parte, isolados dessa gestão. 


\section{Dificuldades em criar um ambiente de trabalho seguro e saudável}

O ambiente de trabalho, abrangendo aspectos físicos, psicossociais e organizacionais, age tanto direta quanto indiretamente sobre a saúde do trabalhador: diretamente ao provocar ou impedir danos ao trabalhador, isto é, interferir sobre o seu estado de saúde; indiretamente ao influenciar a capacidade do trabalhador de lidar efetivamente com as demandas e desafios das atividades laborais, cuidar de sua saúde e manter os seus recursos pessoais. Um ambiente de trabalho seguro e saudável protege os trabalhadores de ameaças à sua saúde e ao mesmo tempo amplia a capacidade para o trabalho e a produtividade (31).

Além do ambiente físico, diversos fatores organizacionais e psicossociais interferem negativamente sobre o ambiente de trabalho. Lundstrom et al. (30) relacionaram vários deles com impacto negativo sobre a segurança e a saúde dos trabalhadores dos serviços de saúde. Esses fatores incluem ambiguidade e conflitos relativos a atribuições, sobrecarga, trabalho monótono, pressão no trabalho, medo de perder o emprego, desconforto físico e falta de suporte social, de controle sobre o trabalho e de participação nas decisões, resultando em insatisfação, esgotamento, alta rotatividade, doenças e acidentes ocupacionais, saúde mental reduzida, depressão e até mesmo suicídio. Trabalho em turnos (utilizado para permitir a continuidade do trabalho na empresa além da carga diária de cada trabalhador), assim como trabalho noturno ou em horários irregulares, também foram prejudiciais à saúde dos trabalhadores (32).

No Brasil, estudos com profissionais de enfermagem identificaram fatores negativos semelhantes: sobrecarga de trabalho, dificuldade em delimitar os diferentes papéis, problemas nas relações interpessoais, carga emocional, recursos inadequados e falta de poder de decisão e de reconhecimento (33). Outros, como as condições precárias de trabalho e o achatamento salarial, associados a más condições de vida, agravam ainda mais a situação (34), sujeitando os profissionais a manter mais de um vínculo de trabalho e a uma carga mensal longa e desgastante $(33,34)$. Tais situações negativas normalmente decorrem da estrutura e da organização do serviço, que no setor saúde ainda sofrem forte influência do modelo taylorista/fordista, da adminis- tração clássica e do modelo burocrático (35). Assim, os serviços são pautados pela lógica da autoridade legal, por hierarquias verticais, com responsabilidades e linhas de mando fragmentadas, pela formalização das relações, pela centralização de programas e de normas de atendimento e pela quase ausência de comunicação tanto entre os serviços em relação horizontal de poder quanto entre os distintos níveis hierárquicos $(35,36)$.

Esse modelo acarreta uma intensa divisão técnica do trabalho, com separação entre concepção e execução, e um grande contingente de trabalhadores semiqualificados, desmotivados e alienados, com baixa possibilidade de ampliar sua capacidade de se comprometer com outros e de intervir de forma autônoma no processo de trabalho $(3,35,36)$. Como a troca de informações e a participação significativa dos trabalhadores são necessárias durante o processo de formação de um ambiente de trabalho saudável $(27,37)$, então o modelo organizacional normalmente adotado em serviços de saúde pode se constituir em uma barreira significativa para as intervenções que tenham esse objetivo.

O compromisso dos gestores seria uma das condições necessárias à criação de um ambiente de trabalho seguro e saudável $(30,38)$. Assim, as dificuldades em se obter um compromisso genuíno seriam limitantes do processo, pois o foco primário das organizações é a produção, não a segurança e a saúde (38). Uma das sugestões desses autores seria a intensificação das ações do poder público, por meio de fiscalização, intervenções e outras ações punitivas e persuasivas, deslocando o foco um pouco mais para a criação e a manutenção desses ambientes. Outra forma de aumentar o compromisso dos gestores seria demonstrar, de forma clara e inequívoca, a correlação entre um ambiente de trabalho seguro e saudável e uma melhora dos resultados ou da satisfação dos clientes. Nos Estados Unidos, os magnet hospitals - instituições credenciadas pela Associação Americana de Enfermeiros, nas quais a qualidade da assistência aos pacientes e a satisfação profissional dos enfermeiros são elevadas, entre outros critérios - oferecem mais segurança e saúde tanto para os trabalhadores quanto para os pacientes, com menor mortalidade destes (30).

Um outro fator limitante é a dificuldade de estabelecer e reconhecer uma correlação clara entre situações no am- biente de trabalho e danos à saúde dos trabalhadores. Isso ocorre principalmente quando a etiologia é multifatorial e há longos períodos de latência entre a exposição e o dano. Nessas circunstâncias, pode parecer que os problemas de saúde decorrem unicamente de características individuais e que só podem ser abordados por meio de iniciativas também individuais (38), não dirigidas à modificação do ambiente.

Os programas inconsistentes de vigilância da saúde do trabalhador prejudicam o estabelecimento dessa correlação entre os riscos e as condições ambientais e os danos à saúde, pois dificultam a identificação dos determinantes negativos sobre a saúde dos trabalhadores e das ações necessárias para alterar o ambiente de trabalho. No Brasil, o dimensionamento real dos danos à saúde dos trabalhadores é bastante prejudicado pela grande subnotificação dos agravos e pelo viés dado ao fluxo oficial de informações, cujo objetivo é o pagamento de benefícios previdenciários e não a investigação dos fatores que contribuem para os agravos (39). Um outro aspecto é que essa vigilância deveria abranger também as doenças e os problemas de saúde não relacionados diretamente com o trabalho, já que qualquer prejuízo à saúde dos trabalhadores pode diminuir a capacidade para o trabalho e possivelmente também impactar a formação de ambientes seguros e saudáveis. Fischer et al. (34) relatam que certas condições do ambiente de trabalho, como desconforto térmico, abuso verbal contra o trabalhador e organização do local de trabalho, além de aspectos sociodemográficos e relacionados à saúde individual dos trabalhadores, correlacionaram-se a um índice de mais de $20 \%$ de profissionais da enfermagem com capacidade inadequada para o trabalho em um hospital universitário em São Paulo, ilustrando a necessidade de os programas de vigilância em saúde do trabalhador irem além dos acidentes e doenças ocupacionais diagnosticados e notificados.

A falta de correlação clara entre as condições do ambiente de trabalho e os danos à saúde também prejudica a concessão de poder de controle sobre o ambiente aos trabalhadores, principalmente os de níveis hierárquicos inferiores (38). Um estudo em hospital público na Bahia constatou menor poder de controle entre auxiliares de enfermagem quando comparados aos enfermeiros da mesma insti- 
tuição (40). O menor poder de controle no trabalho também pode ser uma barreira à formação de um ambiente seguro e saudável, além de diretamente prejudicar a saúde dos trabalhadores, aumentando seus níveis de estresse $(33,41)$ ou resultando em impactos negativos sobre sua saúde mental (40).

$O$ engajamento das lideranças sindicais com a segurança e a saúde dos trabalhadores é essencial para a criação de um ambiente de trabalho mais seguro e saudável (38). No Brasil, o envolvimento dos sindicatos foi fundamental para mudar a regulamentação de segurança e saúde no trabalho, reconhecer as doenças profissionais, criar serviços e programas de saúde do trabalhador no serviço público de saúde (SUS) e melhorar os serviços de medicina ocupacional dentro das indústrias (42). Portanto, a inexistência de representações sindicais fortes (27), o enfraquecimento das existentes, como o que vem ocorrendo em consequência das recentes mudanças econômicas (3), ou uma atuação sindical restrita a outras questões, como as salariais $(38,42)$, podem representar uma barreira adicional às intervenções para melhorar os ambientes de trabalho.

A percepção dos trabalhadores sobre as ações e políticas relacionadas a uma melhoria no ambiente de trabalho também pode ser limitante do processo. Um exemplo é a crença de que certas ações trarão consequências negativas para a carreira ou o avanço no emprego, como no caso das políticas de horários flexíveis e de redução de jornada para apoio à família (38). O prejuízo pode de fato ocorrer se os trabalhadores beneficiários dessas políticas são preteridos pelo empregador quando há oportunidades de avanços no emprego ou na carreira, justificando assim a percepção dos trabalhadores. Também há relatos sobre medo de demissão como causa para não notificar o acidente (43), o que atrapalha o planejamento de ações para tornar os ambientes mais seguros.

Em serviços de saúde, há mais uma particularidade relacionada à percepção dos trabalhadores, relativa às atividades de cuidados a outras pessoas. Em relatos de trabalhadores, por vezes a interferência nos cuidados ao paciente é referida como razão para não adotar as medidas de precaução padrão $(18,28)$. Também há evidências de que o conflito entre o objetivo de tratar e a real possibilidade de fazê-lo pode contribuir para o esgotamento e prejudicar o engajamento nas atividades laborais, afetando especialmente os profissionais da enfermagem (44). Assim, essa característica também deve ser considerada no planejamento de ações para a melhoria dos ambientes de trabalho em questão.

\section{CONSIDERAÇÕES FINAIS}

Ao planejar e realizar intervenções voltadas à prevenção de acidentes ou doenças ou à promoção da saúde dos trabalhadores no setor saúde, é recomendável observar se há participação dos trabalhadores e dos sindicatos, um modelo teórico válido para guiar as ações, métodos e instrumentos apropriados para medir os resultados em curto, médio e longo prazos, integração da intervenção com o contexto do desenvolvimento organizacional e com os projetos de reorganização, canais para comunicação facilitada entre os participantes, comunicação dos resultados obtidos e transferência do conhecimento obtido a partir da intervenção (37). Esses elementos têm contribuído para o sucesso de tais intervenções.

Por outro lado, a ausência dos itens acima listados prejudica a obtenção de resultados satisfatórios a partir das intervenções realizadas. Resumidamente, essas barreiras seriam as seguintes: programas de intervenção sem boa base teórica e não integrados à gestão do serviço como um todo; falhas em avaliar a eficácia das intervenções; restrição das ações de vigilância a doenças e agravos específicos; falta de compromisso da gestão com as intervenções; falhas na comunicação; falta de participação e controle dos trabalhadores sobre o ambiente de trabalho; e programas e intervenções baseados exclusivamente na mudança comportamental dos trabalhadores.

De todas essas barreiras, a mais importante é a falta de integração dos programas dirigidos à saúde do trabalhador com as atividades de gestão. Se tais programas não forem inseparáveis e indistinguíveis da gestão dos serviços de saúde, permanecerão fragmentados, com pouco alcance e resultados inferiores aos possíveis. Além disso, a integração dos programas à gestão induz a um maior compromisso dos gestores com sua execução bem-sucedida, sendo ambos esses aspectos condições que ajudam a transpor os demais obstáculos.

Finalizando, outro elemento norteador para a formulação de programas e intervenções apropriados é a existência de uma legislação que atenda às necessidades do setor. Já se observam as primeiras iniciativas nesse sentido no Brasil. Neste país, à NR-32 e às alterações na legislação previdenciária e da saúde vem-se somar a Política Nacional de Segurança e Saúde do Trabalhador, que teve sua minuta publicada em maio de 2005 (45). Sua ênfase em ações integradas e interinstitucionais, abrangendo todos os trabalhadores, independentemente da forma de vinculação ao trabalho e privilegiando as ações de promoção, prevenção e proteção sobre as de reparação, certamente poderá contribuir para a melhoria da saúde dos trabalhadores da saúde.

\section{REFERÊNCIAS}

1. Santana VS. Saúde do trabalhador no Brasil: pesquisa na pós-graduação. Rev Saude Publica. 2006;40(spe):101-11.

2. Greco DB, Castro Neto M, Tupinambás U. O caso da AIDS: epidemia da doença, epidemia do medo e seus subprodutos. Em: Buschinelli JTP, Rocha LE, Rigotto RM, eds. Isto é trabalho de gente? Vida, doença e trabalho no Brasil. Petrópolis: Editora Vozes; 1994. Pp. 636-72.
3. Deluiz N. Qualificação, competências e certificação: visão do mundo do trabalho. Formação. 2001;1(2):7-17.

4. Organização Pan-Americana da Saúde, Ministério da Saúde. A transformação da gestão de hospitais na América Latina e Caribe. Brasília: OPAS/MS; 2004.

5. Brasil. Portaria MTE ${ }^{\circ} 485$, de 11 de novembro de 2005. Diário Oficial da União 2005. 16 de novembro:80-94 (seção 1). Disponível em: http://www.mte.gov.br/legislacao/normas _regulamentadoras/nr_32.pdf. Acessado em 19 de fevereiro de 2009.

6. Brasil. Portaria no 1339 , de 18 de novembro de 1999. Diário Oficial da União 1979. 19 de novembro:21-9 (seção 1). Disponível em: http://dtr2004.saude.gov.br/susdeaz/ legislacao/legislacao_det.php?co_legis lacao $=40$. Acessado em 13 de fevereiro de 2008. 
7. Brasil. Decreto no 6 042, de 12 de fevereiro de 2007. Diário Oficial da União 2007. 13 de fevereiro:2-22 (seção 1). Disponível em: http:// www.planalto.gov.br/ccivil/ Ato20072010/2007/Decreto/D6042.htm. Acessado em 13 de fevereiro de 2008

8. Xelegati R, Robazzi MLCC, Marziale MHP, Haas VJ. Chemical occupational risks identified by nurses in a hospital environment. Rev Latino-Am Enfermagem. 2006;14(2):214-9.

9. Almeida CAF, Benatti MCC. Exposições ocupacionais por fluidos corpóreos entre trabalhadores da saúde e sua adesão à quimioprofilaxia. Rev Esc Enferm USP. 2007;41(1):120-6.

10. Machado AA, Costa JC, Gir E, Moriya TM, Figueiredo JFC. Risco de infecção pelo vírus da imunodeficiência humana (HIV) em profissionais da saúde. Rev Saude Publica. 1992;26(1):54-6.

11. Murofuse NT, Marziale MHP. Doenças do sistema osteomuscular em trabalhadores de enfermagem. Rev Latino-Am Enfermagem. 2005;13(3):364-73

12. Elias MA, Navarro VL. A relação entre o trabalho, a saúde e as condições de vida: negatividade e positividade no trabalho das profissionais de enfermagem de um hospital escola. Rev Latino-Am Enfermagem. 2006;14(4):517-25.

13. Nascimento Sobrinho CL, Carvalho FM, Bonfim TAS, Cirino CAS, Ferreira IS. Condições de trabalho e saúde dos médicos em Salvador, Brasil. Rev Assoc Med Bras. 2006; 52 (2): $97-102$

14. Chillida MSP, Cocco MIM. Saúde do trabalhador \& terceirização: perfil de trabalhadores de serviço de limpeza hospitalar. Rev LatinoAm Enfermagem. 2004;12(2):271-6.

15. Vilela RAG, Iguti AM, Almeida IM. Culpa da vítima: um modelo para perpetuar a impunidade nos acidentes do trabalho. Cad Saude Publica. 2004;20(2):570-9.

16. Osterman JW. Beyond universal precautions. Can Med Assoc J. 1995;152(7):1051-5.

17. Siegel JD, Rhinehart E, Jackson M, Chiarello L; the Healthcare Infection Control Practices Advisory Committee. Guideline for Isolation Precautions: preventing transmission of infectious agents in healthcare settings. Disponível em: http://www.cdc.gov/ncidod/ dhqp/pdf/guidelines/Isolation2007.pdf. Acessado em 13 de fevereiro de 2008.

18. Balsamo AC, Felli VEA. Estudo sobre os acidentes de trabalho com exposição aos líquidos corporais humanos em trabalhadores da saúde de um hospital universitário. Rev Latino-Am Enfermagem. 2006;14(3):346-53.

19. Monteiro MI, Chillida MSP, Bargas EB. Educação continuada em um serviço terceirizado de limpeza de um hospital universitário. Rev Latino-Am Enfermagem. 2004;12(3):541-8.

20. Nishide VM, Benatti MCC, Alexandre NMC. Ocorrência de acidente do trabalho em uma unidade de terapia intensiva. Rev Latino-Am Enfermagem. 2004;12(2):204-11.

21. Alves RB. Vigilância em saúde do trabalhador e promoção da saúde: aproximações possíveis e desafios. Cad Saude Publica. 2003; 19(1):319-22.

22. Brevidelli MM, Cianciarullo TI. Análise dos acidentes com agulhas em um hospital universitário: situações de ocorrência e tendências. Rev Latino-Am Enfermagem. 2002;10(6): 780-6.

23. Caixeta RB, Barbosa-Branco A. Acidente de trabalho, com material biológico, em profissionais de saúde de hospitais públicos do Distrito Federal, Brasil, 2002/2003. Cad Saude Publica. 2005;21(3):737-46.

24. Lopes MHBM, Moromizato SS, Veiga JFFS. Adesão às medidas de precaução-padrão: relato de experiência. Rev Latino-Am Enfermagem. 1999;7(4):83-8.

25. Neves ZCP, Tipple AFV, Souza ACS, Pereira MS, Melo DS, Ferreira LR. Hand hygiene: the impact of incentive strategies on adherence among healthcare workers from a newborn intensive care unit. Rev Latino-Am Enfermagem. 2006;14(4):546-52.

26. Vaughn TE, McCoy KD, Beekmann SE, Woolson RF, Torner JC, Doebbeling BN. Factors promoting consistent adherence to safe needle precautions among hospital workers. Infect Control Hosp Epidemiol. 2004;25(7): 548-55.

27. Osorio CM, Huet JM, Minayo-Gomez C. Proposição de um método de análise coletiva dos acidentes de trabalho no hospital. Cad Saude Publica. 2005;21(2):517-24.

28. Gammon J, Morgan-Samuel H, Gould D. A review of the evidence for suboptimal compliance of healthcare practitioners to standard/universal infection control precautions. J Clin Nurs. 2008;17(2):157-67.

29. Andrade AC, Sanna MC. Ensino de biossegurança na graduação em enfermagem: uma revisão da literatura. Rev Bras Enferm. 2007; 60(5):569-72.

30. Lundstrom T, Pugliese G, Bartley J, Cox J, Guither C. Organizational and environmental factors that affect worker health and safety and patient outcomes. Am J Infect Control. 2002;30(2):93-106.

31. DeJoy DM, Wilson MG. Organizational health promotion: broadening the horizon of workplace health promotion. Am J Health Promot. 2003;17(5):337-41.

32. Winwood PC, Winefield AH, Lushington K. Work-related fatigue and recovery: the contribution of age, domestic responsibilities and shiftwork. J Adv Nurs. 2006;56(4):438-49.

33. Murofuse NT, Abranches SS, Napoleão AA. Reflexões sobre estresse e burnout e a relação com a enfermagem. Rev Latino-Am Enfermagem. 2005;13(2):255-61.
34. Fischer FM, Borges FNS, Rotenberg L, Latorre MRDO, Soares NS, Rosa PLFS, et al. Work ability of health care shift workers: what matters? Chronobiol Int. 2006;23(6):1165-79.

35. Matos E, Pires D. Teorias administrativas e organização do trabalho: de Taylor aos dias atuais, influências no setor saúde e na enfermagem. Texto Contexto-Enferm. 2006;15(3): 508-14.

36. Campos GWS. O anti-Taylor: sobre a invenção de um método para co-governar instituições de saúde produzindo liberdade e compromisso. Cad Saude Publica. 1998;14(4): 863-70.

37. Brabant LH, Lavoie-Tremblay M, Viens C, Lefrançois L. Engaging health care workers in improving their work environment. J Nurs Manag. 2007;15(3):313-20.

38. Shannon HS, Robson LS, Sale JEM. Creating safer and healthier workplaces: role of organizational factors and job characteristics. Am J Ind Med. 2001;40(3):319-34.

39. Vasconcellos LCF, Ribeiro FSN. Investigação epidemiológica e intervenção sanitária em saúde do trabalhador: o planejamento segundo bases operacionais. Cad Saude $\mathrm{Pu}$ blica. 1997;13(2):269-75.

40. Araújo TM, Aquino E, Menezes G, Santos CO, Aguiar L. Aspectos psicossociais do trabalho e distúrbios psíquicos entre trabalhadoras de enfermagem. Rev Saude Publica. 2003;37(4):424-33.

41. Suominen T, Savikko N, Puukka P, Doran Di, Leino-Kilpi H. Work empowerment as experienced by head nurses. J Nurs Manag. 2005;13(2):147-53

42. Repullo Junior R, Gomes JR. Brazilian union actions for workers' health protection. Sao Paulo Med J. 2005;23(1):24-9.

43. Napoleão AA, Robazzi MLCC, Marziale MHP, Hayashida M. Causas de subnotificação de acidentes do trabalho entre trabalhadores de enfermagem. Rev Latino-Am Enfermagem. 2000;8(3):119-20.

44. Vinje HF, Mittelmark MB. Job engagement's paradoxical role in nurse burnout. Nurs Health Sci. 2007;9(2):107-11.

45. Brasil. Portaria Interministerial MPS/MS/ MTE no 800, de 3 de maio de 2005. Diário Oficial da União 2005. 13 de fevereiro:43-4 (seção 1). Disponível em: http://www81.dataprev. gov.br/sislex/paginas/65/mps-mte-ms / 2005/800.htm. Acessado em 13 de fevereiro de 2008 .

Manuscrito recebido em 24 de março de 2008. Aceito em versão revisada em 24 de junho de 2008. 
ABSTRACT Objective. To search the literature for circumstances that impede injury and disease prevention and other activities intended to improve the health of the health care worker.

Barriers to interventions aimed at promoting the health of health care workers

Methods. The SciELO database was searched for articles published in 1967-2008. This was supplemented by a PubMed search for the period 1950-2008. The following key words were used to identify articles in English, Portuguese, and Spanish: work, health personnel, occupational, risks, diseases, ergonomics, work ability, quality of life, organization, accidents, work conditions, intervention, and administration. Articles on injury and disease prevention and occupational health in a health care setting in Latin America were selected, along with articles focused on health promotion in the health sector.

Results. The following shortcomings were identified: activities lacked a sound theoretical foundation and were not integrated with the health services management; a failure to evaluate the effectiveness of the activity; health surveillance focused solely on a specific disease or injury; management not committed to the proposed activity; miscommunication; inability of workers to participate, or control the work environment; and, programs or efforts that were limited to changing the workers' behaviors. Conclusions. The literature shows that all the barriers identified by this study affect both the health care workers' health as well as their productivity.

Key words Review, health care sector, occupational health, health personnel, Brazil. 